Journal of the Mathematical Society of Japan Vol. 1, No. 3, May, 1951.

\title{
Sur la Théorie du Corps de Classes sur le Corps des Nombres Rationnels
}

\author{
S. Iyanaga et T. Tamagawa
}

La théorie du corps de classes, telle qu'elle a été fondée par notre vénéré Maitre auquel ce Volume est dédié, donne la perspective à toutes les extensions abéliennes d'un corps de nombres algébriques quelconque de degré fini [1]. Mais il y a des faits qui s'échappent encore à la théorie générale, concernant spécialement la théorie du corps de classes sur le corps des rombres rationnels. Nous en indiquerons deux dans ce qui suit.

Dans le $\S 1$ de ce travail, nous donnerons une forme explicite au symbole de restes normiques de Hasse [2] pour les corps circulaires, et paitant, à la correspondance de Chevalley [3] entre le groupe des idèles 1ationnels et le groupe galoisien de l'extension abélienne maximale du corps des rationnels. Dans le $\S 2$, nous généraliseions la théorie classique du genre des corps quadratiques [4], pour le cas des extensions cycliques du corps des rationnels. Nous n'avons pas réussi à étendre ces resultats pour le cas où le corps de base soit un corps de nombres algébriques quelconque: la formulation mème des résultats correspondarts pour ce cas géréral nous semble difficile à trouver.

\section{Notations}

Pendant tout le cours de ce travail rous rous servirons des notations suivantes :

$R:$ le corps des nombres rationnels,

$R^{*}:$ le groupe multiplicatif des éléments $\neq 0$ de $R$, (le signe * sera employé dans le même sens aussi pour les autres corps)

$p:$ un nombre premier rationnel,

$p_{\infty}$ : le diviseur à l'infini de $R$,

$R_{p}:$ le corps des nombres $p$-adiques,

$R_{\infty}=R_{p_{\infty}}:$ le corps des nombres réels,

$J: \quad$ le groupe (multiplicatif) des idèles de $R$,

$P:$ le groape des idèles principaux de $R$,

$a=\left(a_{p}\right):$ un idèle de $R$ avec les composantes p-adiques $a_{p}$,

$A:$ l'extension abélienne maximale de $R$, 
Sur la théorie du corps de classes sur le corps des nombres rationnels 221

$k:$ un corps circulaire, c'est-à-dire, une extension abélienne finie de $R$, $G(k / R):$ le groupe galoisien de $k / R$,

(S) $=G(A / R)$ : le groupe galoisien de $A / R$, topologisé d'après Krull.

\section{$\S 1$ Sur le symbole de restes normiques}

Soient $G$ le groupe multiplicatif de toutes les racines de l'unité, et $G_{p}$ le sous-groupe de $G$ composé de toutes les $p^{\nu}$-ièmes racines de l'unité $(\nu=0,1,2, \ldots)$ pour un $p$ donné. $G$ se décompose alors en produit direct restreint :

$$
G=\underset{p}{I I^{\prime}} G_{p}
$$

où $p$ dans le second membre parcourt tous les diviseurs premiers, excepté celui à l'infini, de $R .{ }^{1{ }^{1}}$

L'extention abélienne maximale $A$ de $R$ s'obtient d'après Kronecker par l'adjonction à $R$ de tous les éléments de $G$. Tout élément $\sigma$ de S $=G(A / R)$ induit donc un automorphisme du groupe $G$, et réciproquement tout automorphisme de $G$ détermine évidemment un élément de $\$ S . G_{p}$ étant des sous-groupes caractéristiques de $G, \sigma$ induit aussi un automorphisme $\sigma_{p}$ de $G_{p}$, et réciproquement, $\sigma_{p}$ pour tous les $p$ déterminent le $\sigma$ d'après (1).

Soit donc $\zeta$ une $p^{2}$-ième racine primitive de l'unité. $\sigma_{p}$ induit alors un at tomorphisme du groupe cyclique $G_{p, \nu}=\left\{1, \zeta, \zeta^{2} \ldots \zeta^{p^{2}-1}\right\}$, et ox aura $\zeta^{\sigma} p=\zeta^{u} p, \nu, u_{p, \nu}$ étant un entier rationnel premier à $p$. Il est clair que l'on a $u_{p, \nu+1} \equiv u_{p, \nu}\left(\bmod . p^{\nu}\right)$, de soite que $u_{p, \nu}$ converge vers une limite $p$-adique $u_{p}$, quand $\nu$ tend vers l'infini. $u_{p}$ est une unité $p$-adique, car $u_{p, 1}$ est premier à $p$. Cette unité $p$-adique $u_{p}$ caractérise complètement l'automorphisme $\sigma_{p}$, car $G_{p}$ s'interprète comme la limite inductive de $G_{p, \nu}$. Nous écrirons $\sigma_{p}$ $=\varphi_{p}\left(u_{p}\right)$.

Désignons par $U_{p}$ le groupe multiplicatif des unités $p$-adiques, et posons

$$
U_{0}=I_{p}^{\prime} U_{p},
$$

le symbole II désignant cette fois le produit direct complet.

1) Le symbole $\Pi$ désignera tantôt le produit des nombres, le produit direct des groupes tantôt restreint, tantôt complet. L'accent au symbole $\Pi^{\prime}$ signifiera toujours que $\not p$ parcourt seulement les diviseurs premiers finis. 
$U_{p}$ tant topologisé $p$-adiquement, $U_{0}$ est aussi muni de la topologie du pro it direct. On a a'ors

Proposition $I \quad U_{0}$ est comme groupe topologique isomodphe à $\mathbb{S}$.

La démo:sstration est immédiate par ce qui précècle. A $u=I I^{\prime} u_{p} \in U_{0}$ cc : spond $\sigma=I I^{\prime} \sigma_{p}$, avec les $\sigma_{p}=\varphi_{p}\left(u_{p}\right)$.

Nous désignerons par $\varphi_{0}$ l'application isomorphe de $U_{0}$ surr (S) donnée par cette Proposition.

Examinons maintenant la structure di groupe des idèles $J$. Désignons par $U_{\infty}$ le groupe multiplicatif des nombres positifs de $R_{\infty}$ et posons $U$ $=U_{0} \times U_{\infty}$. Ce groupe $U$ peut être identifié avec un sous-groupe de $J$. Les élément.s de ce sous-groupe $U$ seront appelés les idèles unitaires. On a $J=P . U$ par le Théorème fonclamental de l'arithmétique élémentaire.

Et, puisqu'on a évidemment $P \cap U=(1)$, nous avons de plus

$$
J=P \times U=I^{P} \times U_{10} \times U_{\infty},
$$

de sorte que tout élément $a \in J$ s'exprime uniquement en forme

$$
a=u . u_{0} u_{\infty}
$$

avec $\alpha \in P, u_{0} \in U_{0}, u_{\infty} \in U_{\infty}$.

Nous poserons

$$
\varphi(a)=\varphi_{0}\left(u_{0}\right)
$$

où $u_{0}$ est déterminé d'après $(2),\left(2^{\prime}\right) . \varphi$ est alors une application homomorphe du groupe $J$ sur $\left(\$ 3\right.$, le noyau de l'homomorphisme étant $P \times U_{\infty}$.

Soit $a_{p}$ un élém $\approx n t$ de $R_{p}{ }^{*}$. $a_{p}$ peut être identifié avec l'élément de $J$ qui a seulc composante non-neutre $a_{j}$. Cet élément de $J$ se représente aussi par $a_{p}$.

Soit, d'autre part, $k$ un coips circulaire, et $G(k / R)$ le groupe galoisien correspondant. L'homomorphisme naturel de $(3)$ sur $G(k / R)$ se désignera par $\Theta_{k}$. $\Theta_{k} \sigma$ pour $\sigma \in \mathbb{B}$ sera donc un automorphisme de $k / R$.

Théorme $I$ On a la formule

$$
\Theta_{i} \varphi\left(a_{p}\right)=\left(\frac{a_{p}, k}{p}\right)
$$

le second membre étant le symbole de restes normiques de Hasse. 
Démonstration Tous les deux membres de (4) représentent des applications homomorphes de $R_{p}$ * dans $G(k / R)$, et satisfassent à la "formule du pioduit ' :

$$
\operatorname{II}_{p} \Theta_{k} \varphi\left(a_{p}\right)=I_{p}\left(\frac{a_{p}, k}{p}\right)=1
$$

po:ir $\Pi a_{p}=\alpha \in P$.

Or, on sait que le symbole de Hasse se caratérise comme l'application homomorphe de $R_{p}{ }^{*}$ dans $G(k / R)$ pour tous les $p$, satisfaisant à $(5)$ pour laquelle on a, à un nombre fini de $p$ près, la formule

$$
\left(\frac{a_{p}, k}{p}\right)=\left(\frac{k}{p}\right)^{-\nu}
$$

où $\left(\frac{k}{p}\right)$ est le symbole de Aitin, et $\nu$ désigne l'entier de soite que l'on ait $p^{-\nu} a_{p} \in U_{p}$.

Il n'y a donc qu'a vérifier que $\Theta_{k} \varphi$ jouit des mêmes propriétés. Comme il est clair que $\Theta_{k} \varphi\left(\varepsilon_{p}\right)=1$ pour $\varepsilon_{p} \in \dot{U}$, d'après la définition même de $\varphi$, on a seulemert à montrer que

$$
\theta_{k} \varphi(\bar{p})=\left(\frac{k}{p}\right)^{-1}
$$

pour un $\not p \in R_{p}{ }^{*}$ avec $p^{-1} \cdot \bar{p} \in U_{p}$, et cela à un nombre fini de $p$ près.

Prenons un nombre naturel $m$ assez grand pour qu'on ait $R(\zeta) \supset k, \zeta$ étant une $m$-ième racine primitive de l'unité. On peut alors supposer $R(\zeta)$ $=k$ sans perdre la généralité. Dans ces conditions, nous vérifierons (6) pour $p$ non diviseurs de $m$. $\left(\frac{k}{p}\right)$ est alors l'automorphisme $\left(\zeta \rightarrow \zeta^{p}\right)$. Il n'y a donc qu'a faire voir que $\varphi(\bar{p})$ opéré sur $\zeta^{p}$ donne $\zeta$.

Soit $q^{\mu}$ un facteur primaire de $m$. $\zeta$ comme un éléme dnte $G$, se décompose d'après (1) en forme

$$
\zeta=\zeta_{q} \ldots \text { avec } \zeta_{q} \in G_{q}
$$

et l'effet de $\varphi(\bar{p})$ sur $\zeta$ se détermine par ceux de $\varphi(\bar{p})$ sur $\zeta_{q}$. Comme $\bar{p} \in R_{p}{ }^{*}$ et $p \neq q, \bar{p}$ est en effet une unité $q$-adique, et puisque $p \in P$, on a de plus $\varphi(\bar{p})=\varphi\left(\bar{p} p^{-1}\right), \varphi_{q}(\bar{p})=\varphi_{q}\left(\bar{p} p^{-1}\right)=\varphi_{q}\left(p^{-1}\right)$, d'où il est aisé à voir que 


$$
\left(\zeta_{q}^{p}\right)^{\varphi q\left(p^{-1}\right)}=\zeta_{q},
$$

et enfin $\left(\zeta^{p}\right)^{\varphi(\bar{p})}=\zeta$ c. q. f. d.

D'après ce Théorème, on serait en droit d'écrire

$$
\varphi\left(a_{p}\right)=\left(\frac{a_{p}, A}{p}\right)
$$

Si l'on introduit dans $J$ la topologie naturelle (voir Weil [5]), $P, U_{0}$, $U_{\infty}$ forment des sous-groupes fermés de $J, P$ discret, $U_{0}$ compact, $U_{\infty}$ localement compact, et $J$ devient le produit topologique de ces trois groupes. $\varphi$ donne un homomorphisme continu de $J$ sur $\mathbb{S}$, et si $J \ni a=\left(a_{p}\right)$, le produit infini $I I \varphi\left(a_{p}\right)$ converge dans $\mathbb{S}$, et $=\varphi(a)$. En vertu du Théorème précédant, il est clair qu'on a

Théorème 2 L'application $\varphi$ définie par (3) donne la correspondance de Chevalley entre $J$ et (S)'.

\section{$\$ 2$ Sur la theorie du genre}

Considérons d'abord un corps circulaire arbitraire $k$. Les gioupes des idèles et des idèles principaux de $k$ seront désignés respectivement par $J_{k}$, $P_{k}$. On connait ce que signifie la norme $N_{k / k}\left(J_{k}\right)=N_{k}$ de $J_{k}$ dans $R$, et que $P V_{k}$ forme un sous-groupe d'indice $(k: R)$ de $J$. A tout sous-groupe $H$ de $J$ d'indice fini contenant $P$ correspond un corps circulaire $k$, cle sorte q:ie $H=P V_{k}$, et $\theta_{k} \varphi$ du Théorème 1 donne l'isomorphisme entre $G(k / R)$ et $J / P N_{k}$.

D'autre part, la décomposition (2) du groupe $J$ indique qu'à tout sous-groupe $H$ de $J$ d'indice fini contenant $P$ coriespond biunivoquement un sous-groupe $H_{0}$ d'indice fini de $U_{0}$, par les felations: $H \rightarrow H \cap U_{0}$ $=H_{0}, H_{0} \rightarrow H_{0} P=H$.

A tout corps circulaire $k$ correspond doinc un sous-groupe d'indice fini de $U_{0}$, que nous écrirons $H_{0}(k)$. Il est facile à voir, que le degré . de ramification $e_{p}$ de $p$ dans $k$ est donné par

$$
e_{p}=\left(U_{p}: H_{0}(k) \cap U_{p}\right)
$$

2) Après la rédaction de ce travail, M. Iwasawa nous écrit de Princeton, qu'il connait aussi ce résultat. Nos résultats étant d'ailleurs tellement simples, que nous doutons s'ils ne sont tous connus des connaisseurs de la théorie des idèles. Aussi nous ne pré:endon pas à notre priorité en publiant ces résultats. Nous les publions pour la commodité des chercheurs. 
On appelle "différentiels de $R$ " les caractères de $J$ qui annulent $P \times U_{\infty}$. Un différentiel $\chi$ se détermine évidemment par sa contraction $\bar{\chi}$ sur $U_{0}$, et tout caractère $\bar{\chi}$ de $U_{0}$ s'étend à un différentiel. Soient $\chi$ un différentiel, $\bar{\chi}_{p}$ le caractèı de $U_{0}$ ayant la même contraction que $\chi$ sur $U_{p}$, et de solte que $\bar{\chi}_{p}\left(\varepsilon_{q}\right)=1$ pour tout $\varepsilon_{q} \varepsilon U_{q}, q \neq p$. Soit enfin $\chi_{p}$ le différentiel extention de $\bar{\chi}_{p}$. On a alors évidemment

$$
\chi=I_{p}^{\prime} \chi_{p}
$$

et le degré de ramification $e_{p}$ de $p$ coincide avec l'ordre de $\chi_{p}$.

Soit $Z$ l'extension cyclique correspondant à $\chi$. Le degré $(Z: R)$ est alors égal au p.p.c.m. des $e_{p}$. Soient $J_{Z}, P_{Z}, U_{Z}$ les groupes des idèles principaux, des idèles unitaires de $Z$ respectivement. Les ideles unitaires de $Z$ sont les idèles, dont tous les composantes sort des unités p-adiques, où $\mathfrak{p}$ sont les diviseurs de $Z$. Ceci est clair quand $\mathfrak{p}$ est un diviseur fini ; quand $\mathfrak{p}$ est à l'infini, et $Z$ est complexe, tout élément non nul de $Z_{p}$ s'appelle une unité $\mathfrak{p}$--idique, mais quand $Z$ est réel, il convient d'appeler ainsi seuls les éléments positifs de $Z_{p}$. Le groupe quotient $J_{Z} / P_{Z} U_{Z}$ est alors isomorphe au groupes des classes d'idéaux au sens étroit de $Z$, et dans le corps des rationrels, $J / P N_{Z ! n}\left(U_{Z}\right)$ est isomorphe au groupe quotient $R^{*} /($ le groupe des restes normiques modulo le conducteur de $Z / k$ ).

Dans ce qui suit, nous désignerons les éléments de $J_{Z}$ par $\mathfrak{a}, \mathfrak{b}, \ldots$. Le symbole $N$ signifiera toujours la norme de $Z$ à $R$ sauf avis contraire, $\sigma$ désignera un élément générateur du groupe $G(Z / R)$. Les deux Propositions suivantes sont des traductions faciles des faits connus en langage des idèles.

Proposition 2 (Lemme de Hiibe1t) Si $N(\mathfrak{a})=1$, il existe un idèle $\mathfrak{b}$ de sorte qu'on ait $\left.\mathfrak{a}=\mathfrak{b}^{1-\sigma 3}\right)$

Proposition 3 (Lemme de Hasse) On a la formule :

$$
N\left(J_{Z}\right) \cap P=N\left(P_{Z}\right)^{4)}
$$

De ces Propositions s'ensuit le Théorème suivant.

Théorème $3 \mathrm{Si}$

3) Hilbert 1.c. Satz 90. La démonstration de cette Proposition se réduit immédiatement au cas où $\mathfrak{a}=\dot{a}_{\mathfrak{p}} \in k_{\mathfrak{p}} *$. Il faut prendre une précaution spéciale, d'ailleurs facile, au cas où $\mathfrak{p}$ se décompose dans $Z$. Nous laissons au lecteur le soin de compléter la démonstration,

4) Voir Chevalley 1.c. p. 409. 


$$
N_{\mathfrak{a}} \in P N\left(U_{Z}\right),
$$

il existent des idèles $\mathfrak{b}, \mathfrak{u} \in U_{Z}$, et $\alpha_{Z} \in P_{Z}$ de soite qu'on ait

$$
\mathfrak{a}=\mathfrak{b}^{1-\sigma} \mathfrak{u} \alpha_{\boldsymbol{Z}}
$$

Démonstration Par l'hypothèse nous pouvons écrire $N \mathfrak{a}=\alpha N \mathfrak{u}$ avec $u \in P, \mathfrak{u} \in U_{Z}$. En vertu de la Proposition 3 , on a $\alpha=N\left(\mathfrak{a} u^{-1}\right)=N \varkappa_{z}$ avec $u_{Z} \in P_{Z}$, et par suite, par la Proposition 2, $\mathfrak{a l t}^{-1}{\alpha_{Z}}^{-1}=\mathfrak{b}^{1-a}$, c. q. f. d.

Il va sans dire que de (10) s'ensuit (9). Les idèles de $J_{z}$, pour lesquels (9) ou (10) soit vérifié, forment un sous-groupe $H_{Z}$ de $J_{Z}$. Nous appellerons ce sous-groupe $H_{\%}$ le gcnre principal de $Z$, et les éléments du groupe quotient $J_{Z} / H_{Z}$ les genrcs de $Z$.

Cette définition et le Théorème 3 montrent deux choses. $1^{\circ}$ On a

$$
J_{Z} \supset H_{Z} \supset P_{Z} U_{Z} \text {, }
$$

et le nombre des genres $g=\left(J_{Z}: H_{Z}\right)$ est égal au nombre des éléments $C$ du groupe quotient $J_{Z} / P_{Z} U_{Z}$, pour lesquels on a $C^{1-\sigma}=1$. On appelle ces éléments $C$ les classes ambiges. $2^{3}$ Quand on applique homomorphiquement $J$, dans $J / P$ par la formation de la norme, $J_{z}$ tombe sur $P N\left(J_{z}\right)$ et $H_{Z}$ sur $P N\left(U_{Z}\right)$. On a donc

$$
J_{Z} / H_{Z} \cong P V\left(J_{Z}\right) / P N\left(U_{Z}\right) .
$$

Le Théorème suivant donne une information exacte sur le nombre $g$.

Thiorime 4 On a la formule

$$
g=\frac{I^{\prime} c_{p}}{n},
$$

où $n=(Z: R)$.

Démonstration On sait que $\left(J: P N\left(J_{Z}\right)\right)=n$ d'après un résultat fondamental de la théorie du corps de classes. Puisqu'on a d'après (11)

$$
\begin{aligned}
g & =\left(J_{Z}: H_{Z}\right)=\left(J N\left(J_{Z}\right): P N\left(U_{Z}\right)\right) \\
& =\left(J: P N\left(U_{Z}\right)\right) /\left(J: P N\left(J_{Z}\right)\right) \\
& =\left(J: P N\left(U_{Z}\right)\right) / n,
\end{aligned}
$$

on n'a qu'à montrer

$$
\left(J: P N\left(U_{Z}\right)\right)=\underset{p}{I}{ }^{\prime} e_{p}
$$


Sur la théoric du corps de classes sur le corps des nombres rationnels 227

Or, comme nous avons fait remarquer au début de ce paragraphe, le groupe quotient $J / P N\left(U_{z}\right)$ peut itre " transféré" sur $U_{0} / N\left(U_{z}\right)$ en prenant l'intersection avec $U_{0}$. (Ici on fait usage de rotre convention sur $U_{Z}$ concernant les diviseurs à l'infini pour avoir $\left.N\left(U_{Z}\right) \subset U_{0}\right)$. O.l a par conséquent

$$
\begin{aligned}
\left(J: P N \text { A }\left(U_{Z}\right)\right) & =\left(U_{0}: N\left(U_{Z}\right)\right) \\
& =I_{p}^{\prime}\left(U_{p}: N\left(U_{,}\right) \cap U_{p}\right) \\
& =I_{p}^{\prime}\left(U_{p}: H_{0}(Z) \cap U_{p}\right)=I_{p}^{\prime} e_{p} . \quad \text { c.q.f.d. }
\end{aligned}
$$

Une condition nécessaire et suffisante pour qu'un éément a de $J$ soit contenu dans $P N\left(U_{Z}\right)$ est donnée par

$$
\chi_{p}(\mathfrak{a})=1 \quad \text { pour tous les } p .
$$

D'autre part, a est contenu dans $P \dot{N}\left(J_{Z}\right)$, si et seulement si

$$
\operatorname{II}_{i} \chi_{p_{i}}(\mathfrak{a})=1
$$

où $p_{i}$ sont des rombres premiers pour lesquels $\chi_{p_{i}} \neq 1,(i=1,2, \ldots, h)$.

Par conséquent, si $\zeta$ sont des racines $e_{h}$-ièmes arbitrairement données de l'unité satisfaisant à la seule condition $\prod_{i=1}^{h} \zeta_{i}=1$, on peut trouver $\mathfrak{a} \epsilon J_{\zeta}$ de sorte qu'on ait

$$
\chi_{p_{i}}(N \mathfrak{a})=\zeta_{i} . \quad i=1,2, \ldots, h,
$$

et le vecteur $\left(\zeta_{1}, \ldots, \zeta_{h}\right)$ dépend seulement aı genre auquel a appartient. Si l'on appelle ce vecteur le système de caractères du genre, on peut énoncer le Théorème suivant.

Théorème 5 Les genres de l'extension cyclique $Z$ de $R$ se caractérisent complètement par leurs systèmes de caractères.

\section{Bibliographie}

[1] T. Takagi : Ueber eine Theorie des relativ Abelschen Zahlkörpers, Journ. Coll. Sc. Tokyo 41 (1920).

[2] II. ILasse: Die Normenrestheorie relativ Abelscher Zahlkörper als Klassenkörpertheorie im Kleinen, Crelles Journ. 162 (1930).

[3] C. Chevalley: Ia thérie du corps de classes, Ann。 of Math. 41 (19\%0)

[4] Voir D. Hilbert, Zahlhericht.

[5] A. Weil: Sur la théerie du corps des classes, dans ce même volume, 